\title{
Indication for preoperative localization of small peripheral pulmonary nodules in thoracoscopic surgery
}

\author{
Hajime Saito, MDa \\ Yoshihiro Minamiya, MD \\ Ikuo Matsuzaki, MDa \\ Kasumi Tozawa, MDa \\ Kousei Taguchi, MD \\ Taku Nakagawa, MDa \\ Manabu Hashimoto, $\mathrm{MD}^{\mathrm{b}}$ \\ Yoshinori Hirano, $\mathrm{MD}^{\mathrm{b}}$ \\ Jun-ichi Ogawa, MDa
}

Objective: Widespread clinical use of helical computed tomography has improved the detection rate for small peripheral pulmonary nodules. As a result, use of thoracoscopic surgery to confirm the diagnosis of small peripheral pulmonary nodules has become more important than ever before. However, if small peripheral pulmonary nodules are too small or located too deeply to detect thoracoscopically, it is necessary to mark the small peripheral pulmonary nodules preoperatively. The purpose of this study was to determine indications for preoperative hookwire marking in thoracoscopic resection of small peripheral pulmonary nodules.

Methods: A total of 120 patients underwent thoracoscopic pulmonary resection in our institute from 1999 to 2001. Small peripheral pulmonary nodules were marked preoperatively in 61 of these patients by means of percutaneous placement of a hookwire under computed tomographic guidance. The hookwire-marked small peripheral pulmonary nodules either were smaller than $10 \mathrm{~mm}$ or were located more than $10 \mathrm{~mm}$ below the pleural surface.

Results: Although 9 of the hookwire-marked small peripheral pulmonary nodules were easily identified during thoracoscopy, the other 52 small peripheral pulmonary nodules could not have been identified during thoracoscopy without the hookwire marking. Of the 59 small peripheral pulmonary nodules that were not hookwire marked, 7 required conversion to thoracotomy to locate the nodules. Small peripheral pulmonary nodules from both groups were examined and assigned to either an undetectable or detectable group. Discriminant function analysis indicated that a linear function (ie, depth $=0.836 \times$ size -2.811 ) could be used to differentiate between undetectable and detectable small peripheral pulmonary nodules, and preoperative hookwire marking for small peripheral pulmonary nodules should be considered for nodules in the region above those.

Conclusion: The results suggest that this formula might serve as an indication for preoperative marking of small peripheral pulmonary nodules in thoracoscopic resection.

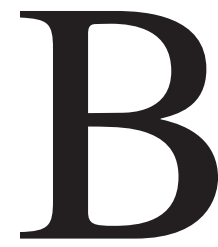

ecause of the recent developments in radiologic diagnosis with helical computed tomography (CT), small peripheral pulmonary nodules (SPPNs) that were previously undetectable by means of conventional CT can now be detected. ${ }^{1}$ As a result of advances in diagnostic technology, the role of video-assisted thoracoscopic surgery (VATS) in thoracic disease has expanded.2,3 VATS is used in diagnostic excisional biopsies of SPPNs that could not be successfully diagnosed by means of bronchoscopic or transthoracic needle biopsy and also in therapeutic 
resections of peripheral lung cancer. ${ }^{4}$ A major limiting factor in the success of thoracoscopic resection has been the difficulty in locating small or deeply situated target nodules. Therefore, a nodule judged too small or located too deeply beneath the pleural surface to be seen or palpated during thoracoscopy must be localized preoperatively. Several techniques have been used to locate SPPNs before thoracoscopy. These include CT-guided percutaneous hookwire placement, ${ }^{5,6}$ metallic coil injection, ${ }^{7}$ indicator dye injection, ${ }^{8,9}$ colored collagen injection, ${ }^{10}$ and intraoperative ultrasonographic guidance. ${ }^{11}$ Although all of the aforementioned methods are effective in locating the target lesion, indications for preoperative localization with these methods have not been clarified.

In this study a group of 120 patients undergoing VATS wedge resection of peripheral pulmonary nodules was reviewed in which preoperative hookwire placement was used for nodules smaller than $1 \mathrm{~cm}$ in size or greater that $10 \mathrm{~mm}$ from the pleural surface. We performed a retrospective review to clarify the utility of preoperative hookwire placement for nodule identification and developed a mathematic equation relating depth and size of the nodule on the basis of the preoperative computed tomographic findings.

\section{Methods}

From January 1999 through December 2001, 120 patients (62 men and 58 women) whose ages ranged age from 22 to 83 years (median age, 61 years) underwent partial resection of the lung by using thoracoscopy in our institute. Although biopsy during fiberoptic bronchoscopy was done preoperatively, all patients also underwent chest computed tomographic examination that revealed indeterminate pulmonary nodules. The purpose of the thoracoscopic resection was to obtain a histopathologic diagnosis to exclude or confirm the presence of primary or secondary malignant lesions.

The chest computed tomographic scan was carefully examined before the operation to determine whether the nodule could be identified by its location at the time of thoracoscopy. On the basis of the previous experience, we applied our criteria for preoperative localization according to the preoperative computed tomographic findings. If the nodule was pleural or subpleural in location and 1 $\mathrm{cm}$ or larger, it was assumed that the nodule would be detectable by means of visual inspection or instrument palpation at the time of exploratory thoracoscopy. In this case hookwire placement is not necessary. For nodules deeper than $1 \mathrm{~cm}$ below the pleural surface or less than $1 \mathrm{~cm}$ in size, preoperative localization was used to identify the location of the nodule and improve the success of the thoracoscopic resection. Hookwire placement under CT was performed in 61 patients with small, deeply situated, or both peripheral pulmonary lesions requiring thoracoscopic resection. Under computed tomographic guidance, a 20-gauge needle from a localizer system (VATS marker, Hakko Medical) was placed percutaneously through the chest wall into the pulmonary nodule. The route to the nodule was selected to navigate through the chest wall and avoid vital intrathoracic structures, to traverse the shortest possible transpulmonary distance, and to enter the lung nearly perpendicularly to the pleural surface. Once optimal placement of the localizing needle tip was confirmed by means of computed tomographic scanning, a hookwire inserted through the localizing needle was placed adjacent to the target nodule. It is very important to have the patient suspend respiration during the insertion of the hookwire. The localizing needle was then withdrawn, and the external end of the wire was looped widely and taped to the chest with sterile tape. The wire should contain a wide loop because the hookwire might dislocate by means of respiration or pneumothorax. Computed tomographic examination was again performed in a 2-mm scan to verify placement of the localizing hookwire before the patient was transported to the operating room for thoracoscopic resection.

Thoracoscopic surgery was performed after achievement of general anesthesia with a double-lumen endotracheal tube for single-lung ventilation. The lung of the pneumothorax side is blocked by means of single-lung ventilation immediately after intubation to avoid tension pneumothorax. The patient was placed in the lateral decubitus position, with the trocar placed in the midaxillary line in the seventh intercostal space. General exploratory thoracoscopy was performed to determine the location of the target pulmonary nodule. An additional intercostal incision was made under direct vision on the anterior or posterior line to manipulate the target nodule. At least 3 of the authors who are thoracic surgeons tried to identify the nodule. When the nodule was not visible by means of thoracoscopy or not palpable with thoracoscopic instruments, the index finger of the surgeon was placed through one of the trocar sites to palpate the surface of lung. If the nodule can not be identified by using the above method, thoracoscopic surgery was converted to a minithoracotomy. Once the nodule was identified, a thoracoscopic wedge resection was performed. When the localizing hookwire had been placed before the operation, the nodule's location could be easily determined at the time of thoracoscopy. If the hookwire had become inadvertently dislodged, the subtle subpleural hematoma that usually occurs at the entry site of the needle could be identified. After resection of the small pulmonary nodule, histologic examination was immediately performed on all specimens. If the nodule was a benign tumor histologically, the operation was finished after insertion of a chest tube. When the nodule was histologic (ie, a primary malignant tumor), VATS lobectomy and lymph node dissection was performed.

All patients were examined and assigned to either the undetectable or detectable group. Nodules were categorized according to size of the nodule and depth from the pleural surface.

Values are expressed as means \pm SD. Significant differences were assessed by means of $t$ tests or discriminant analysis between the undetectable and detectable groups.

\section{Results}

\section{Background of Patients}

A total of 120 patients underwent partial resection of the lung by means of thoracoscopy (Table 1). Because difficulty in detection can be anticipated when nodules are more than $1 \mathrm{~cm}$ below the pleural surface or less than $1 \mathrm{~cm}$ in size, preoperative localization is required. Preoperative localization of pulmonary nodules was performed on $61(50.8 \%)$ 
TABLE 1. Background of patients who underwent partial resection of SPPNs during thoracoscopy

\begin{tabular}{lcc}
\hline & $\begin{array}{c}\text { Nonpreoperative } \\
\text { marking }\end{array}$ & $\begin{array}{c}\text { Preoperative } \\
\text { marking } \\
\text { (hookwire) }\end{array}$ \\
\hline No. & 59 & 61 \\
Age (y) & $61.0 \pm 13.0$ & $60.0 \pm 12.0$ \\
Size (mm) & $15.4 \pm 5.7$ & $7.8 \pm 2.9$ \\
Depth from pleura (mm) & $5.3 \pm 4.5$ & $10.0 \pm 7.5$ \\
\hline
\end{tabular}

\section{TABLE 2. Definitive diagnoses for all patients}

\begin{tabular}{lc}
\hline Primary lung cancer & \\
$\quad$ Adenocarcinoma & $71(59.2 \%)$ \\
Squamous cell carcinoma & $2(1.7 \%)$ \\
Small cell carcinoma & $1(0.8 \%)$ \\
Metastatic lung tumor & $16(13.3 \%)$ \\
Carcinoid tumor & $2(1.7 \%)$ \\
Atypical adenomatous hyperplasia & $2(1.7 \%)$ \\
Inflammation & $7(5.8 \%)$ \\
Tuberculosis & $6(5.0 \%)$ \\
Granuloma & $6(5.0 \%)$ \\
Lymph apparatus & $4(3.3 \%)$ \\
Eosinophilic tumor & $1(0.8 \%)$ \\
Hamartoma & $1(0.8 \%)$ \\
Leiomyoma & $1(0.8 \%)$ \\
\hline
\end{tabular}

patients in whom the maximal nodule size was $7.8 \pm 2.9$ $\mathrm{mm}$ or the depth from the pleura was $10.0 \pm 7.5 \mathrm{~mm}$. Thoracoscopic surgery without preoperative localization was performed on $59(49.2 \%)$ patients in whom nodule size was $15.4 \pm 5.7 \mathrm{~mm}$, depth from the pleura was $5.3 \pm 4.5$ $\mathrm{mm}$, or both.

\section{Pathologic Diagnosis of Small Pulmonary Nodules}

A definitive diagnosis was obtained in all patients (Table 2). Malignant disease accounted for $76.7 \%$, including adenocarcinoma in $71(59.2 \%)$ patients, squamous cell carcinoma in $2(1.7 \%)$ patients, small cell carcinoma in $1(0.8 \%)$ patient, metastatic lung tumor in $16(13.3 \%)$ patients, and carcinoid tumor in $2(1.7 \%)$ patients. Bronchioloalveolar cell carcinoma accounted for 45\% (32/71 patients) of adenocarcinomas. The other nodules were the result of benign diseases (23.3\%), including atypical adenomatous hyperplasia in $2(1.7 \%)$ patients.

\section{Conversion to Thoracotomy in the Nonpreoperative Marking Group}

A total of 13 (22.0\%) patients in the nonpreoperative marking group (Table 3 ) required conversion to thoracotomy. Inability to locate the nodule was the reason in 7 (53.8\%) patients.

\section{Analysis of Factors for Localizing Small Nodules} Fifty-two of the 61 nodules preoperatively localized with a hookwire could not have been identified without the preop-
TABLE 3. Reasons for conversion to thorarcotomy in the nonpreoperative marking group

\begin{tabular}{ll}
\hline Inability to localize the nodule & $7(53.8 \%)$ \\
Adhesion & $5(38.5 \%)$ \\
Large resection area & $1(7.7 \%)$ \\
\hline
\end{tabular}

TABLE 4. Size and depth of SPPNs in the detectable and undetectable groups

\begin{tabular}{lccc}
\hline & Detectable & Undetectable & $\boldsymbol{P}$ value \\
\hline No. & 61 & 59 & \\
Size $(\mathrm{mm})$ & $14.9 \pm 5.9$ & $8.4 \pm 3.8$ & $<.0001$ \\
Depth $(\mathrm{mm})$ & $2.3 \pm 2.6$ & $12.0 \pm 6.5$ & $<.0001$ \\
\hline
\end{tabular}

erative marking. Nine of the preoperatively marked patients did not require hookwire localization to identify the wedge resection. On the other hand, of the 59 thoracoscopic lung resections performed without hookwire localization, 7 patients required conversion to thoracotomy caused by the inability to locate the nodule. All nodules were examined, classified as either detectable or undetectable, and categorized according to the size of the nodule and depth from the pleural surface (Table 4). In the 61 cases identified through thoracoscopy, the maximum size of the nodule was $14.9 \pm$ $5.9 \mathrm{~mm}$, with a depth from the pleural surface of $2.3 \pm 2.6$ $\mathrm{mm}$. Both size and depth were significantly different in the undetectable group. These factors were related to the inability to locate the nodules.

Both size and depth factors in each group were mapped. A linear discriminant analysis (Figure 1) indicated that detectable and undetectable nodules could be significantly discriminated by using the following function: depth = $0.836 \times$ size -2.811 . This suggests that preoperative localization of SPPNs should be considered when the nodule lies in the region above the function. This formula might serve as an indication for preoperative localization of SPPNs in thoracoscopic resection.

\section{Discussion}

In this study we examined SPPNs identified by means of thoracoscopy or by means of preoperative localization. All cases were analyzed according to nodule size and depth from the pleural surface. A linear function (ie, depth = $0.836 \times$ size -2.811 ) could provide reliable discrimination between the detectable and undetectable groups. This formula might serve as an indication of need for preoperative localization of SPPNs in thoracoscopic resection.

In recent years, the indications and capabilities of thoracoscopy have been broadened as a result of improved videooptics and surgical instrumentation..$^{2,3}$ Recent developments with helical CT have increased the preoperative diagnostic ability to detect SPPNs, including localized bronchioloal- 


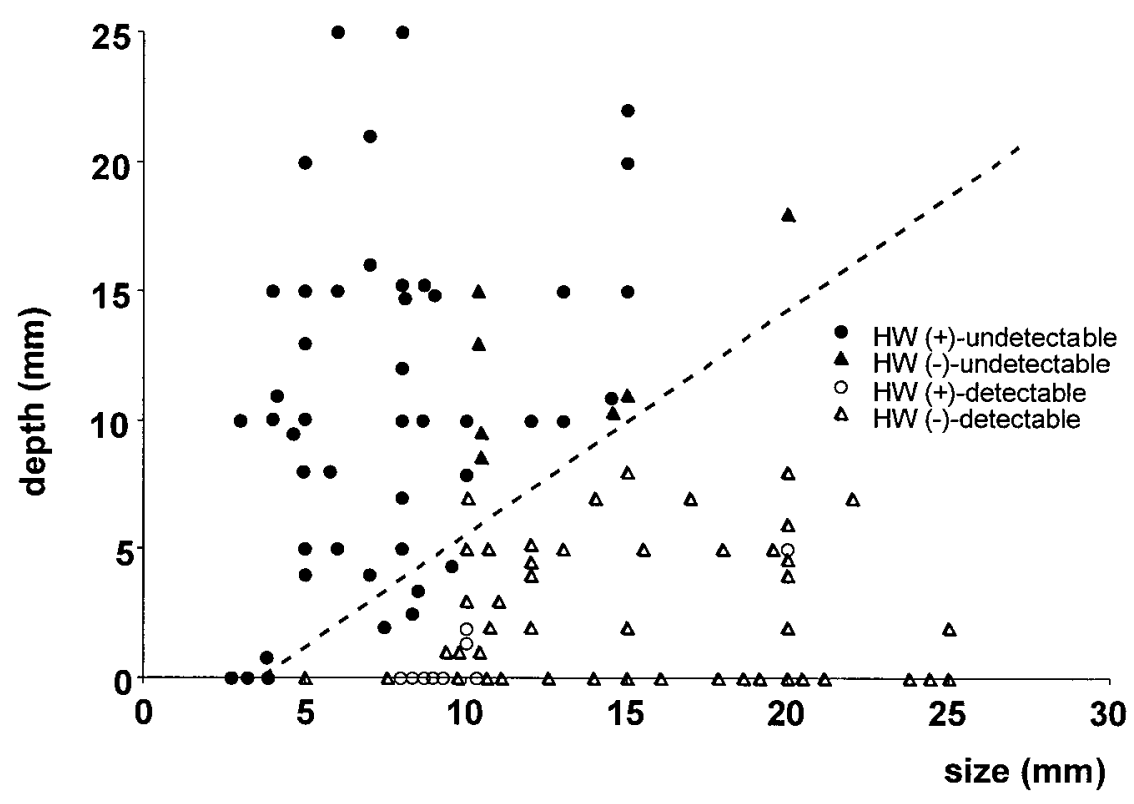

Figure 1. Fifty-two of 61 nodules marked by preoperative hookwire localization could not have been identified without the preoperative marking (filled circles). Nine patients did not require hookwire localization to identify the wedge resection (open circles). Of 59 thoracoscopic lung resections without hookwire localization, 52 nodules could be identified by means of thoracoscopy alone (open triangles). In 7 patients the procedure was converted to thoracotomy because of the inability to locate the nodules (filled triangles). Factors such as size and depth were mapped in each group. All 120 patients were classified into detectable or undetectable groups. By means of discriminant analysis, nodules were evaluated with respect to size and depth from the pleural surface. The results indicate that a linear function (ie, depth $=0.836 \times$ size -2.811 ) can be used to reliably separate detectable and undetectable groups. HW, Hookwire.

veolar carcinoma. ${ }^{1}$ The role of thoracoscopic surgery as a means of minimally invasive surgery has now expanded for diagnostic, as well as therapeutic, excision when transbronchial or transthoracic needle aspiration biopsy fails to yield a definite diagnosis of benign disease in an indeterminate SPPN. ${ }^{4}$

One of the limiting factors in thoracoscopic resection of the lung is the inability to determine the exact location of a target pulmonary nodule that is small or located far below the pleural surface. If judged to be too small or too deep beneath the pleural surface to be seen or palpated during thoracoscopy, the nodule must be localized preoperatively. Any effective method for locating the SPPNs preoperatively can identify the target lesion. Percutaneous localization of SPPNs with a hookwire under computed tomographic guidance has been performed in our institute. Asamura and colleagues $^{7}$ summarized the advantages of hookwire placement as follows: (1) the exact location of the nodule and the depth from the pleural surface can be identified by lifting the marker; (2) a safe and sufficient surgical margin can be ensured if the resection line is far enough away from the mark; and (3) even minithoracotomy for palpation to determine the location of a nodule can be avoided. On the other hand, possible complications might include bleeding with hematoma formation or tension pneumothorax. Bleeding can be avoided by means of careful insertion of a hookwire under computed tomographic imaging so that the hookwire does not injure the larger vessels. Mild pneumothorax can occur if there is no pleural adhesion to the chest wall. Hookwire placement is performed within 1 hour before induction of general anesthesia with a double-lumen endotracheal tube. The lung of the pneumothorax side is blocked by means of single-lung ventilation immediately after intubation, thus avoiding severe tension pneumothorax that would require emergency chest drainage before the operation.

Preoperative localization appears to improve the likelihood of successful wedge resection of some SPPNs, but the procedure itself entails significant additional invasion that can cause procedural complications. Although the concept of preoperative localization is to avoid procedures that are more invasive, there are few reports promoting less invasive preoperative localization. This decision not to use preoperative localization has apparently been based on the experience of the surgeons. ${ }^{12,13}$ Suzuki and coworkers ${ }^{13}$ reported that distance from the pleural surface is very important in 
predicting visibility. If a lesion is less than $10 \mathrm{~mm}$ in size, the probability that the nodule can not be located increases if the distance to the pleural surface is greater than $5 \mathrm{~mm}$. These findings are fairly consistent with the statistical significance demonstrated in this article with respect to separating the detectable and undetectable groups according to the following function: depth $=0.836 \times$ size -2.811 .

If the nodule is solid and large enough, endoscopic visual identification might be possible because pulmonary nodules bulge against the surrounding atelectatic lung. Palpation with intrathoracic instruments is the first means of searching whether the nodule cannot be identified visually. However, some types of nodules, such as localized bronchioloalveolar carcinoma, are difficult to locate. Even if the nodule is large, close to the pleural surface, and palpable with the surgeon's finger through the intercostal access, its character is very soft, small, faint, and of similar consistency to the surrounding normal lung parenchyma. Localized bronchioloalveolar carcinoma shows a replacement growth of atypical cells with mild thickening of the alveolar septa, sometimes without fibrotic foci. ${ }^{14}$ Pleural indentation or spiculation is usually lacking in this type of tumor. Because it would be very difficult to locate the tumor, even through intraoperative digital palpation, preoperative localization is thought to be necessary for this type of tumor.

In the present study our objective was to retrospectively identify indications for preoperative localization. Our results were based on 120 patients who underwent thoracoscopic pulmonary resection. All cases were classified as to whether the nodule could be identified by means of thoracoscopy alone (detectable) or whether preoperative localization was required (undetectable). Nodules were analyzed for size and depth from pleural surface. A linear function (ie, depth $=0.836 \times$ size -2.811 ) was found to be statistically significant in separating the detectable from the undetectable groups. This formula might therefore be used as an indication for preoperative localization of SPPNs in thoracoscopic resection.

We thank Dr Kawamoto, technical research laboratory, Kyowa Hakko Kogyo Company, for his technical support in statistical analyses. We also thank Ms Mitsuko Sato and Jun Kodama for their secretarial support.

\section{References}

1. Kaneko M, Eguchi K, Ohmatsu H, Kakinuma R, Naruke T, Suemasu $\mathrm{K}$, et al. Peripheral lung cancer: screening and detection with low-dose spiral CT versus radiography. Radiology. 1996;201:798-802.

2. Mack MJ, Aronoff RJ, Acuff TE, Douthit MB, Bowman RT, Ryan WH. Present role of thoracoscopy in the diagnosis and treatment of disease of the chest. Ann Thorac Surg. 1992;54:403-9.

3. Landreneau RJ, Hazelrigg SR, Ferson PF, Johnson JA, Nawarawong W, Boley TM, et al. Thoracoscopic resection of 85 pulmonary lesion. Ann Thorac Surg. 1992;54:415-20.

4. Allen MS, Deschamps C, Lee RE, Trastek VF, Daly RC, Pairolero PC. Video-assisted thoracoscopic stapled wedge excision for indeterminate pulmonary nodule. J Thorac Cardiovasc Surg. 1993;106:104852.

5. Shepard JO, Mathisen DJ, Muse VV, Bhalla M, McLoud TC. Needle localization of peripheral lung nodules for video-assisted thoracoscopic surgery. Chest. 1994;105:1559-63.

6. Shah RM, Spirn PW, Salazar AM, Steiner RM, Cohn HE, Solit RW, et al. Localization of peripheral pulmonary nodules for thoracoscopic excision: value of CT-guided wire placement. AJR Am J Roentgenol. $1993 ; 161: 279-83$

7. Asamura H, Kondo H, Naruke T, Tsuchiya R, Wakao F, Kaneko M, et al. Computed tomography-guided coil injection and thoracoscopic pulmonary resection under roentgenographic fluoroscopy. Ann Thorac Surg. 1994;58:1542-4.

8. Moon S, Wang Y, Jo K, Kwack M, Kim S, Kwon O, et al. Fluoroscopy-aided thoracoscopic resection of pulmonary nodule localized with contrast media. Ann Thorac Surg. 1999;68:1815-20.

9. Lenglinger FX, Schwarz CD, Artmann W. Localization of pulmonary nodules before thoracoscopic surgery: value of percutaneous staining with methylene blue. AJR Am J Roentgenol. 1994;163:297-300.

10. Nomori $\mathrm{H}$, Horio $\mathrm{H}$. Colored collagen is a long-lasting point marker for small pulmonary nodules in thoracoscopic operation. Ann Thorac Surg. 1996;61:1070-3.

11. Greenfield AL, Steiner RM, Liu JB, Cohn HE, Goldberg BB, Rawool $\mathrm{NM}$, et al. Sonographic guidance for the localization of peripheral pulmonary nodules during thoracoscopy. AJR Am J Roentgenol. 1997; 168:1057-60.

12. Demmy TL, Wagner-Mann CC, James MA, Curtis JJ, Schmaltz RA, Walls JT. Feasibility of mathematical models to predict success in video-assisted thoracic surgery lung nodule excision. Am J Surg. 1997; 174:20-3.

13. Suzuki K, Nagai K, Yoshida J, Ohmatsu H, Takahashi K, Nishimura $\mathrm{M}$, et al. Video-assisted thoracoscopic surgery for small indeterminate pulmonary nodules. Chest. 1999;115:563-8.

14. Noguchi M, Morikawa A, Kawasaki M, Matsuno Y, Yamada T, Hirohashi S, et al. Small adenocarcinoma of the lung: histologic characteristics and prognosis. Cancer. 1995;75:2844-52. 